\title{
Biowaiver for Immediate and Modified Release Dosage forms Scientific summary of the CSPS workshop
}

\author{
Raimar Löbenberg ${ }^{1}$, Karen Reynolds ${ }^{2}$, Krishnan Tirunellai ${ }^{2}$, Emilija Fredro-Kumbaradzi ${ }^{3}$, Robin \\ Zhang $^{2}$ Kimberly Raines ${ }^{4}$, Mathi Mathivanan ${ }^{5}$, Barbara M Davit ${ }^{6}$, Anita Laloo ${ }^{6}$, Shrinivas Murti ${ }^{6}$, \\ Shereeni Veerasingham ${ }^{2}$, Laszlo Endrenyi ${ }^{7}$, Fakhreddin Jamali $^{1}$ \\ ${ }^{1}$ Faculty of Pharmacy and Pharmaceutical Sciences, University of Alberta; ${ }^{2}$ Bureau of Pharmaceutical Sciences, \\ Therapeutics Products Directorate, Health Canada; ${ }^{3}$ Apotex Inc. Canada; ${ }^{4}$ FDA, Center for Drug Evaluation and \\ Research/Office of Pharmaceutical Quality/ Office of New Drug Products/Division of Biopharmaceutics III; \\ ${ }^{5}$ Teva Canada; ${ }^{6}$ Merck \& Co. USA; ${ }^{7}$ University of Toronto, Leslie Dan Faculty of Pharmacy
}

Correspondence author: Raimar Löbenberg, Faculty of Pharmacy \& Pharmaceutical Sciences, University of Alberta, 11361 - 87 Avenue NW, Edmonton, Alberta, Canada

T6G 2E1; Raimar@ualberta.ca

\begin{abstract}
A joint Canadian Society for Pharmaceutical Sciences and Health Canada workshop entitled "Biowaiver for Immediate and Modified Release Dosage forms" was held in Ottawa, November $19^{\text {th }} 2015$. A summary of all presentations is included.
\end{abstract}

\author{
Abbreviations \\ AADA, Antibiotic Drug Application; \\ ANDA, Abbreviated New Drug Application; \\ API, Active Pharmaceutical Ingredient; \\ BA, Bioavailability; \\ BCS, Biopharmaceutics Drug Classification \\ System; \\ BDDCS, Biopharmaceutics Drug Disposition \\ Classification system; \\ BE, Bioequivalence; \\ b.i.d., bis in die (twice a day); \\ BP, British Pharmacopeia; \\ BPS, Bureau of Pharmaceutical Sciences; \\ Cmax, Maximum Plasma concentration; \\ CMC, Chemistry and Manufacturing Controls; \\ CQA, Critical Quality Attribute; \\ CRF, Code of Federal Regulations; \\ CRP, Canadian Reference Product; \\ DESI, Drug Efficacy Study Implementation; \\ EMA, European Medicines Agency; \\ ER, Extended Release \\ FDA, U.S. Food and Drug Administration; \\ FDC, Fixed Drug Combinations; \\ GIT, Gastrointestinal tract;
}

\author{
IR, Immediate Release; \\ IVIVC, in vitro/in vivo correlation; \\ MCC, Micro Crystalline Cellulose, \\ MR, Modified Release; \\ NDA, New Drug Application; \\ NOC, Notice Of Compliance; \\ NSAIDS, Non-Steroidal Anti-Inflammatory Drugs; \\ NTI, Narrow Therapeutic Drug; \\ PD, Pharmaceutical development; \\ PK, Pharmacokinetic; \\ Q\&A, Questions and Answers; \\ QbD, Quality by Design; \\ QC, Quality Control; \\ q.d., quaque die (one a day); \\ rpm, Rounds per minute; \\ SAC-PSCP, Scientific Advisory Committee on \\ Pharmaceutical Sciences and Clinical \\ Pharmacology; \\ SmPC, Summary of Product Characteristics; \\ SUPAC, Scale-up and Post Approval Changes; \\ Tmax, Time to maximum plasma concentration; \\ TPD, Therapeutic Products Directorate; \\ USP, United States Pharmacopeia; \\ WHO, World Health Organization
}

The workshop was opened by Karen Reynolds the Director of the Bureau of Pharmaceutical Sciences (BPS) of the Therapeutic Products Directorate (TPD), Health Canada. She pointed out the importance of workshops for the regulator, industry and other stakeholders. "It will be important to discuss areas where regulators, the industry and other stakeholders agree and the areas where both disagree."

The workshop proceeded by the following presentations: 


\section{Desired state: Opportunities and Challenges}

Krishnan Tirunellai, Ph. D., Bureau of Pharmaceutical Sciences, Therapeutics Products Directorate, Health Canada.

Sponsors might have various reasons including multiple strengths of the drug product (solid orals), changes made during product lifecycle during pre-approval (clinical trial phase) and post-approval phase [1] For example, if the manufacturing or processing is changed at the manufacturing site.

To obtain a Biowaiver is very important to companies because of the substantial savings in resources and time. However, if not done with scientific justification it could impact safety/efficacy due to false positive decisions. Often differences in bioavailability are not detected in medical practice and hence seldom reported.

Biowaiver issues have led to numerous challenges for both regulators and industry. Industry might relay too much on dissolution test results to make critical decisions, which could lead to false negative (recalls) or false positive results (adverse impact on safety/efficacy).

Unfortunately, regulators show a lack of a convergence due to different or not comprehensive guidance documents. Important aspects of guidelines are not based on science but rather on a risk-based approach. e.g., SUPAC or Health Canada's proportionality table. [2]

When comparing requirements from other agencies one should also look at their additional requirements on quality, like pre-approval product-specific inspection to gain complete confidence on information provided in the premarket submission. Agencies might use of science \& risk-based Quality by Design approaches. Sometimes additional requirements e.g., commercial lots for use of non-standard manufacturing methods will be requested. Others perform complete testing of all imported drug product lots. The above requirements provide a much-desired assurance to support product approval decisions.

TPD's post-NOC guidance is based on Risk-based approach. [2] It covers common but not all scenarios that could qualify for biowaiver based on in vitro results. [2] However, it is the company's responsibility to evaluate the risk when it is not clear though the guidance. The company should contact TPD in writing to seek additional clarification. For example, Modified Release dosage forms are considered to be of higher risk. Significant changes e.g. change in composition, manufacturing site or process etc. would need supporting biostudies or and IVIVC. Here the link between quality (product, process and controls) with clinical outcomes (i.e., pharmacokinetics, safety and efficacy) have to be established. All drugs should not be treated alike. Considerations should go beyond non-linear PK and NTI drugs. Tools like the Proportionality table for oral IR or MR products is arbitrary. [2] Here fixed numbers are used for low risk products (e.g., BCS class I and III or highly soluble APIs). Changes should be based on scientific justification with statistical design. More attention should be paid to product development (e.g., QbD type). Dissolution test results should be used mainly for confirmation or as additional proof of concept that is presented in the dossier, and not as the main evidence in the decision-making process. f2 statistic values should be used very carefully in the decision making process with the exception when an IVIVC is used. [3] The severity of risk (false or positive) posed by a poorly soluble drug does not change. However, science and technology could be used in a meaningful manner to minimise the probability of occurrence and mitigate the residual risk.

A poor choice for process and test methods can contribute to an increase in the overall risk of making a wrong decision. In contrast, science and improved product development can help in making a better-informed decision.

In conclusion, there are opportunities for biowaivers when science- and risk-based approaches are used to develop products. This will ensure good quality products reach the patients. Such approaches would facilitate the regulatory approval process and minimise market uncertainties such as shortages and recalls. Today there is more transparency and collaboration between Health Canada with 
other regulatory agencies and industry. An open dialogue would help Health Canada in developing a better guidance and convergence that would be beneficial for industry and the regulator.

\section{Biowaivers: Challenges and Opportunities, Generic Industry Perspective}

Emilija Fredro-Kumbaradzi, Ph.D., Apotex Inc.

The rate and extent of absorption is controlled by dissolution as a formulation factor and solubility and permeability as drug substance parameters. If the predefined criteria for these three factors are met, a biowaiver can be granted. Biowaivers can be requested for different purposes. Under certain conditions, a biowaiver can be granted based on BCS class of a drug, [4] or alternatively, non-BCS based biowaivers, applicable to all BCS classes, can be granted in lieu of justifying compositions changes, process changes or for line of strengths. In a case of biowaivers, Bioequivalence between reference and test product is established in vitro, instead of in vivo. [5] This should not be misunderstood as "no" equivalence test performed.

Industry faces some challenges in regard to non-BCS based biowaivers. For example, for fixed drug combinations, formulation proportionality as a prerequisite for a biowaiver is not always given and hence can be interpreted in different ways. Another challenge are multifunctional excipients and interpretation of their role in formulation. In vitro performance of the product can also pose obstacles, examples of enteric coated products and the impact of tablet size and disintegration times were discussed.

\section{Fixed drug combinations (FDC)}

FDC products contain two or more drug substances in a single dosage form. The active drug substances can be in the same (single) compartment or in a separate (multilayer) compartments. What are the criteria to assess proportionality?

In a single compartment:

What is the role of the second active?

What is the \% change of the second active?

What is the total additive effect of the \% change (active + excipient)?

In a multi compartment system:

Is the proportionality based on the layer or the entire unit? products:

Here are the examples of different guidelines on proportionality of fixed drug combination

Canada: "Bioequivalence of proportional formulations [6]: - does not cover proportionality of combinations.

The U.S.: "Draft Guidance BA and BE Submitted in NDAs or INDs" [7]:

Bilayer tablets are considered to be one formulation even though they consist of two separate layers with different compositions. In assessing the proportional similarity of the different strengths, all components of both layers should be proportionally similar (whole tablet). Exceptions to the above definitions may be possible if adequate justification is provided.

The E.U.: Guideline for the investigation of the bioequivalence[8]:

The conditions regarding proportional composition should be fulfilled for all active substances of fixed combinations. When considering the amount of each active substance in a fixed combination the other 
active substance(s) can be considered as excipients. In the case of bilayer tablets, each layer may be considered independently.

The proposed options for proportionality assessment are:

For single compartment products, define $\%$ variability of second active and total $\%$ change allowed.

For separate compartments, assessment of the proportionality for the whole tablet or as individual compartments may result in different interpretation of formulation proportionality and consequently impact the feasibility of a biowaiver request.

However, each approach, in combination with adequate in vitro dissolution similarity, should be considered if supportive of biowaiver justification.

\section{Multifunctional excipients}

Then the issue of multifunctional excipients was discussed. Multifunctionality is a trend and preference in formulation development. Ingredients in composition can have multiple roles - it is important to understand which one is primary function for a particular formulation. The relative amount in a formulation should be considered when defining a primary role.

For example, Micro Crystalline Cellulose (MCC) can be a diluent, binder, disintegrant or flow aid; starch, pregelatinized can be a binder, disintegrant, flow aid, lubricant and/or taste masking agent; Crospovidone can be a disintegrant, binder, dissolution enhancer; Even drug substances with their properties may act as binder or disintegrant.

MCC roles and acceptable variability in formulation:

If $\mathrm{MCC}$ is a diluent it can be changed $\pm 5.0 \%$; here it is considered as an inert bulking agent; however, as a binder it can only be changed by $\pm 0.5 \%$; here it acts via interlocking, forming solid bridges or hydrogen bonds; while as disintegrant MCC can be changed by $\pm 1.0 \%$ and its capillary action is the main function. The different concentrations in formulation are normally associated to its function: Concentration as diluent up to $99 \%$, as binder $5-20 \%$, as disintegrant $5-15 \%$. [2]

What is the expected impact of an increased concentration on the drug release from each functional category? The expected impact as diluent is little to no impact, as binder it might have a negative impact and as disintegrant it might have a positive effect. Evidently, they are all different. Hence primary role should be defined for each formulation.

Crospovidone - roles and acceptable variability:

Its main function can be disintegrant $( \pm 1.0 \%)$ or binder $( \pm 0.5 \%)$. As disintegrant its rapid swelling is the main mechanism as binder its plastic deformability is important.

\section{Regulatory aspects of multifunctional excipients:}

Canada: In the Guidance for Post-Notice of Compliance (NOC) Changes: Quality Document states [2]: ".... a scientific justification for the assigned primary role and the percentage level used in composition is required along with supporting data to demonstrate that the wide range used considering primary function does not affect the auxiliary functions of the multifunctional excipient."

The U.S.: The Q\&A for Scale-up and Post-Approval Changes Guidance for Immediate Release Products (SUPAC-IR) [1]:

"... composition changes are based on being able to define the use or action of the particular excipient in the product.

... if an excipient was utilized to provide multiple functions.... then the most conservative recommended change should be followed

....explanation of how the change will affect other functions of the excipient in the product."

The options for industry arising from these guidance documents are challenging. How to evaluate the acceptable level of change for multifunctional excipient?

What would be sufficient for justifying its role? One way to answer this question can be by applying QbD approach [9] with full product understanding where the main function of the excipient is 
assigned and appropriately justified, followed by an assessment of the impact of the \% change on Critical Quality Attributes; For such studies dissolution similarity will be the key criteria. Finally, a design space can be defined.

\section{Delayed Release products and 2 statistics}

Scenario: the dissolution similarity between the bio-strength and a lower strength has $\mathrm{f} 2<50$. However, the cores are $\mathrm{f} 2$ similar and the coating thickness is similar. Can the lag time be normalized before $\mathrm{f} 2$ calculations as per Japanese Guideline? [10]

\section{Regulatory aspects:}

Canada, US, EU have no specific recommendations for delayed release products

Japan: In the Guidance for Bioequivalence, enteric-coated products are grouped with the immediate release products with the provision of demonstrating acid resistance. Furthermore, dissolution curves can be adjusted for lag times before the assessment of similarity is performed. Lag time is defined as the time when $5 \%$ of the labeled claim dissolves; lag time should be determined by linear interpolation; differences in lag time should not be more than $10 \mathrm{~min}$ in order to qualify for lag time normalization.

The following questions arise from this:

What is the goal of the enteric-coat?

- If its function is to ensure acid resistance and to protect from release in acid; the cores can be considered as an immediate release composition.

- Are small differences in lag time critical in this case?

- How can this be demonstrated?

- What are the additional options to assess similarity?

- Further proof of comparable acid resistance should be demonstrated (several low $\mathrm{pH}$ conditions) or lag time normalization could be applied or both combined?

\section{Issues with disintegration of tablets with different sizes:}

Tablets with different sizes will disintegrate at different rates. This will impact the dissolution rate and f2 similarity between formulation proportional tablets of different size/strength. An example was given for a drug, which has high solubility at lower $\mathrm{pH}$ but low solubility at $\mathrm{pH} 6.8$. Accordingly, the difference in tablet sizes caused the f2 value $<50$ between the lower and higher strength at $\mathrm{pH} 6.8$; however, simulations using a physiological modeling software suggested there is no relevance of $\mathrm{f} 2$ dissimilarity in $\mathrm{pH} 6.8$ on absorption profile. [11]

Possible solutions to similar scenario and approach in assessment of relevance of $\mathrm{f} 2$ dissimilarity can be:

1. Consider drug product exposure to lower $\mathrm{pH}$ values in GIT before reaching $\mathrm{pH} 6.8$ (disintegration occurs at lower $\mathrm{pH}$ ).

2. Physiological modeling might be used to assess compartmental absorption pattern in the GIT. [12]

3. Designing a dynamic dissolution protocol to reflect $\mathrm{pH}$ changes along GIT. [13]

4. Differences and similarities can be assessed on a risk-based approach.

5. Lag time normalization is another option (similar to the Japanese guideline)

In conclusion, Regulatory Guidance's on biowaiver policy are based on scientific understanding of Active Pharmaceutical Ingredient (API) properties and drug product properties in correlation to their in vivo behavior. Industry can utilize $\mathrm{QbD}$ based product development strategies to deepen their understanding of the drug product and scientifically justify biowaiver requests. Integration of scientific knowledge will serve both, agencies and industry, to agree on acceptable biowaiver applications to provide safe and effective drug products. 


\section{An overview of Biowaivers globally}

Raimar Löbenberg, Ph.D., Drug Development and Innovation Centre at the University of Alberta

The introduction of the Biopharmaceutical Drug Classification system (BCS) in 1995 paved the way for the mechanistic evaluation of factors governing bioavailability. [14] The sum of two mechanisms is responsible for the bioavailability of a compound: solubility in the intestine and gut permeability. Only what is dissolved can get absorbed and only what crosses the gut wall can be found in the blood stream. Therefore, drug substances are biopharmaceutically classified by their solubility within the physiological relevant $\mathrm{pH}$ range and small intestinal permeability.

Table 1. BCS classification of APIs [15]

\begin{tabular}{|c|c|c|}
\hline BCS Class & Solubility & Permeability \\
\hline I & High & High \\
\hline II & Low & High \\
\hline III & High & Low \\
\hline IV & Low & High \\
\hline
\end{tabular}

The FDA defined "highly soluble" (as the) highest marketed dose strength(is) soluble in 250 $m l$ of aqueous media over a $\mathrm{pH}$ range of $1-7.5$ at $37^{\circ} \mathrm{C}$

and "highly permeable" when the extent of absorption in humans is determined to be greater or equal to $90 \%$ of an administered dose based on a mass balance determination or in comparison to an intravenous reference dose. [5] Other agencies and guidelines have slightly different definitions and different FDA guidance documents vary in the relevant $\mathrm{pH}$ ranges.

The SUPAC guidance from 1995 was the first Guidance for Industry which allowed to establish bioequivalence using in vitro testing. [1] This was a fundamental change away from clinical bioequivalence studies towards dissolution testing.

Figure 1 shows the different pathways to establish bioequivalence (therapeutic equivalence) of pharmaceutically equivalent products.

The FDA's SUPAC guidance [1] defined three levels of manufacturing changes and the dissolution requirements to demonstrate bioequivalence. Level 1 changes are those that are unlikely to have any detectable impact on formulation quality and performance.

Level 2 changes are those that could have a significant impact on formulation quality and performance.

Tests and filing documentation vary depending on three factors: therapeutic range, solubility, and permeability, see Table 2.

Only Level 1/2 changes are eligible for biowaivers. Level 3 changes, which are likely to have a significant impact on formulation quality and performance need to perform in vivo bioequivalence studies.

In 1997 the FDA issued another guidance: Extended Release Oral Dosage Forms: Development, Evaluation, and Application of In Vitro/In Vivo Correlations. [16]

This guidance provides recommendations to pharmaceutical sponsors who intend to develop documentation in support of an in vitro/in vivo correlation (IVIVC) for an oral extended release (ER) drug product for submission in a:

- new drug application (NDA),

- abbreviated new drug application (ANDA), or

- antibiotic drug application (AADA). 


\section{Bioequivalence}

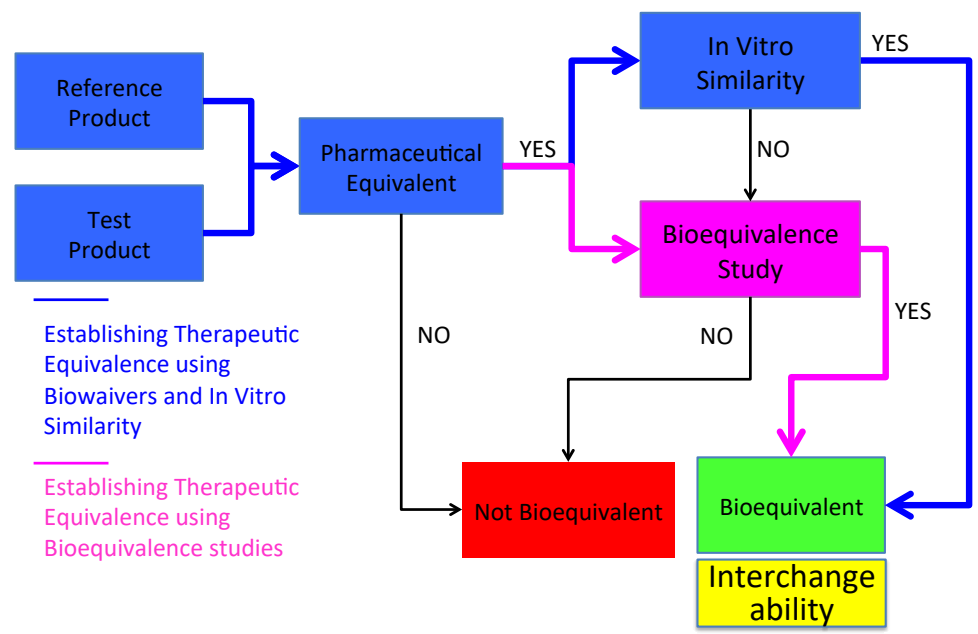

Figure 1: Bioequivalence can be established via in vitro or in vivo studies.

The guidance explains methods for developing an IVIVC and evaluating its predictability and how to set dissolution specifications. The guidance defines an IVIVC as "A predictive mathematical model describing the relationship between an in vitro property of a dosage form (usually the rate and extent of drug dissolution or release) and a relevant in vivo response, e.g., plasma drug concentration or amount of drug absorbed". Only Level A correlations, which are point to point correlations between in vivo and in vitro data, can be used to request a biowaiver. If an IVIVC is established, it can serve as a surrogate for in vivo bioequivalence.

Table 2: SUPAC dissolution criteria according to API properties

\begin{tabular}{|l|l|}
\hline BCS characteristic of the API & Dissolution criterion \\
\hline $\begin{array}{l}\text { High Permeability, } \\
\text { BCS Class 1 }\end{array}$ & Dissolution of $85 \%$ in 15 minutes in $900 \mathrm{~mL}$ of $0.1 \mathrm{~N} \mathrm{HCl}$. \\
\hline $\begin{array}{l}\text { Low Permeability, } \\
\text { High Solubility Drugs } \\
\text { BCS Class 3 }\end{array}$ & $\begin{array}{l}\text { Multi-point dissolution profile should be performed in the application / } \\
\text { compendial medium at } 15,30,45,60 \text { and } 120 \text { minutes or until an asymptote } \\
\text { is reached. }\end{array}$ \\
\hline $\begin{array}{l}\text { High Permeability, } \\
\text { BCS Class 2 }\end{array}$ & $\begin{array}{l}\text { Multi-point dissolution profiles should be performed in water, } 0.1 \mathrm{~N} \mathrm{HCl,} \mathrm{and} \\
\text { USP buffer media at pH } 4.5,6.5, \text { and } 7.5\end{array}$ \\
& $\begin{array}{l}\text { Adequate sampling should be performed at } 15,30,45,60, \text { and } 120 \text { minutes } \\
\text { istil either } 90 \% \text { of drug from the drug product is dissolved or an asymptote }\end{array}$ \\
\hline
\end{tabular}

The FDA [5], WHO [17], Health Canada [4] and EMA [8] have issued and updated their biowaiver guidance documents for immediate release dosage forms based on the BCS classification of the API for immediate release products. While the FDA guidance from 2000 limits biowaivers to BCS class 1 drugs the recent draft guidance from 2015 extends biowaivers to BCS class 3 drugs. [18] 
Additionally, the permeability criterion of $90 \%$ oral absorption was lowered to $85 \%$. However, the volume of the dissolution medium was limited to $500 \mathrm{~mL}$ down from $900 \mathrm{~mL}$. The most significant difference between FDA and other guidance documents is the dose consideration. FDA uses since 1995 the highest strength which is in line with general bioequivalence testing while the EMA, Health Canada and WHO now use the highest therapeutic dose in the $\mathrm{pH}$ range of $1.2-6.8$. The WHO guide from 2006 used like FDA the highest strength and considered BCS class 2 drugs, which are weak acids for biowaivers. This was based on the findings of Yazdanian et al. who demonstrated that NSAIDs fail the BCS 1 classification due to their poor solubility in the stomach. However, if their solubility is measured at $\mathrm{pH}$ 5.0- 7.4 most of them will be highly soluble, at this for drug absorption relevant $\mathrm{pH}$ range. [19, 20] In 2015 the WHO [21] removed the BCS class 2 acids and changed to the highest therapeutic doses instead of highest dose strength. Table 3 summarized the different regulatory criteria.

Table 3: BCS based Biowaiver Guidance criteria

\begin{tabular}{|l|l|l|}
\hline BCS Guidance Document & API properties & BCS criteria \\
\hline FDA 2000 & BCS 1 & $\begin{array}{l}\text { Highest Dose Strength } \\
250 \mathrm{ml} @ \mathrm{pH} 1.2-7.4 \\
>90 \% \text { absorption } \\
>85 \% \text { oral absorption }\end{array}$ \\
\hline EMA & BCS 3 & $\begin{array}{l}\text { Highest Therapeutic Dose } \\
250 \mathrm{ml} @ \mathrm{pH} 1.2-6.8 \\
>85 \% \text { oral absorption }\end{array}$ \\
\hline Health Canada & BCS $1+3$ & $\begin{array}{l}\text { Highest Therapeutic Dose } \\
250 \mathrm{ml} \text { @ pH 1.2-6.8 } \\
>85 \% \text { oral absorption }\end{array}$ \\
\hline WHO 2006 & BCS 1+3 & $\begin{array}{l}\text { Highest Dose Strength } \\
\text { Highest Therapeutic Dose }\end{array}$ \\
WHO 2015 & BCS 1,3,2 (acids only) & $\begin{array}{l}250 \mathrm{ml} @ \text { pH 1.2-6.8 } \\
>85 \% \text { oral absorption }\end{array}$ \\
WHO 2006, 2015 & BCS 1,3 & \multicolumn{2}{|l}{} \\
\hline
\end{tabular}

The obviously small differences in the guidance documents can cause huge challenges for globally operating pharmaceutical companies.

For example amoxicillin is listed on the WHO list of essential medicines. [22] The dose of 500 $\mathrm{mg}$ is classified as a BCS 1 API. $500 \mathrm{mg}$ is soluble in $185 \mathrm{ml}$ of water and has a bioavailability is $89 \%$. EMA classifies amoxicillin as BCS 2 API because their guidance considers the highest single dose and not highest dose strength. Finally, the FDA classified amoxicillin as BCS 4 drug with $90 \%$ or more absorption. This example shows that harmonization is urgently needed. The BCS was introduced to simplify and scientifically classify APIs. The different guidance documents, however, cause more uncertainty due to their differences. [20]

It is important to notice that the BCS classification applies to the API and not to the biopharmaceutical properties of a finished product. However, biowaivers are granted for finished products or dosage forms in development if they contain an API, which is eligible for a biowaiver. If a product fails in vitro similarity testing it might still be therapeutic equivalent when passing a bioequivalence study. Today, biowaivers can be applied to demonstrate bioequivalence between phase $2 / 3$ products, commercial product and bio-batch, approval of products at lower dose strengths, for SUPAC based changes, IVIVC of extended release products and the approval of generics.

Figure 2. shows the current scientific status of biowaivers, which includes weak acids of BCS class 2 /4 APIs. If there is an uncertainty in the permeability classification of a drug the approach 
outlined by the Biopharmaceutics Drug Disposition Classification system (BDDCS) [23] can be helpful. Drugs, which are highly metabolized, can be considered highly permeable. This is due to their similar permeability crossing into enterocytes and hepatocytes.

The FDA issued in 2015 a guidance document for "Dissolution Testing and Specification Criteria for Immediate-Release Solid Oral Dosage Forms Containing Biopharmaceutics Classification System Class 1 and 3 Drugs". [24]

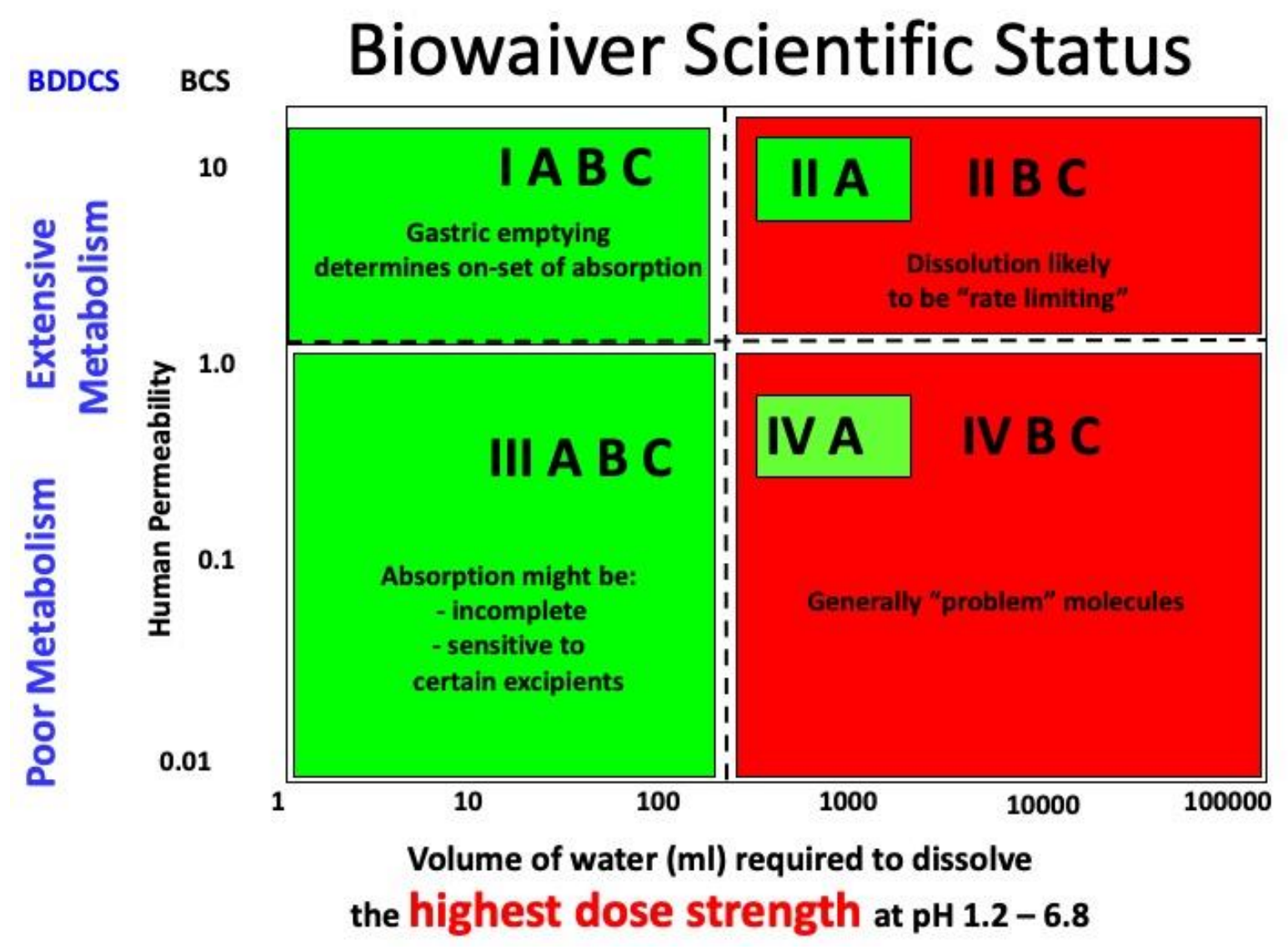

Figure 2: Biowaiver status according to scientific knowledge. The BSC classes can be sub divided into acids (A) bases (B) and neutral molecules (C). The Biopharmaceutics Drug Disposition Classification system (BDDCS) can help to classify drugs using metabolism as additional criterion.

This document allows substituting dissolution with disintegration testing if the release of a drug product is over $85 \%$ in 15 minutes and disintegration is fast within 5 minutes. The problem with this guideline is that these criteria lack mechanistic studies and are purely empirical. A study was presented which differentiated between API driven dissolution and dosage form dependent dissolution. [25] Using such an approach could add valuable mechanistic information and help to justify disintegration or dissolution testing for drug products.

In conclusion, the BCS is a scientific framework assisting in rational mechanistic drug development and to apply for Biowaivers. However, guidance documents differ globally slightly, and this causes confusion and the same drug product might be assigned different BCS classes in different regions of the world. This might allow to apply for a Biowaiver in one country and excludes the same product from a biowaiver in another country. Harmonization is needed to address this situation. 


\title{
Biowaiver for Immediate and Modified Release Dosage forms Biowaiver of Generic Products - Examples and Challenges
}

\author{
Robin Zhang, Ph.D., The Bureau of Pharmaceutical Sciences, Health Canada
}

Biowaivers are requested by companies for various reasons. In the examples he discussed the focus was on biowaivers for additional strengths of tablets/capsules.

The requirements for biowaivers are described in the TPD policy "Bioequivalence of Proportional Formulations: Solid Oral Dosage Forms (1996)" [6]

Some of the common deficiencies Health Canada has observed over the years were discussed and examples were given.

The general observation of the regulator was:

- Although information on pharmaceutical development (PD) in the generic submissions has improved over the years, QbD-type information is rarely submitted.

- Information on PD is patchy and often does not flow in a logical manner.

- Dissolution data is given to support biowaivers but often the summary linking the choice of formulation, and manufacturing method, with the in vitro dissolution data is not submitted.

- Functions of excipients are described in general terms; unique properties that could potentially impact quality and performance are not described.

- Additional (critical) information on PD is not included in the original submission and is made available only when asked by TPD.

- Selected dissolution methodology is often not supported by justification that it is bio-relevant and/or discriminatory. This includes use of surfactants.

- Choice of formulation: When a common blend is not used or when the \% of excipient used in some strengths is different from the bio-lot the scientific reason for the final choice of formulation composition (e.g., failure to achieve a satisfactory product) are not explained.

- When the policy criteria of "proportional formulation" is not met (IR products) submissions still report the formulations as "proportional composition" which creates confusion.

- Differences exceed the limits stipulated in Table 1 of the policy and they are not adequately supported by scientific justification:

- Ideally same proportion should be maintained.

- Table 1 represents a relaxation allowing minor differences.

- The role of multifunctional excipients is not discussed or recognized

- Change/difference in release controlling excipient (MR products) are not adequately supported.

- In absence of in vitro/in vivo Correlation (IVIVC), in vitro dissolution studies are not considered to be an adequate substitute for (in vivo) bioequivalence studies.

- similar dissolution profiles are inadequate to justify "non proportional formulations".

- Comparative dissolution profiles of the biowaiver strengths versus the biolot are submitted only in the proposed (QC) release media. Additional dissolution data in media across the physiological $\mathrm{pH}$ range is not submitted.

- QC release method cannot be presumed to be "discriminatory" or sensitive to differences in product characteristics.

- Comparative dissolution profiles of the biowaiver strengths are performed in different dissolution media (surfactant concentrations) than the one used for biolot. The same dissolution method should be used for ALL strengths to allow meaningful comparison.

- Comparative dissolution data with the Canadian Reference Product (CRP) could be important for PD, it is generally not necessarily relevant in the context of biowaiver (comparative dissolution data against the biolot of the same product line is most relevant).

\section{EXAMPLE 1}


Nifedipine Controlled Release Tablets (osmotic pump technology)

- Two strengths: $30 \mathrm{mg}$ and $60 \mathrm{mg}$ (biolot)

- Cores were directly proportional, but the coating wasn't.

Risks:

- Differences in critical formulation attributes of the coatings, which control diffusion rate of water and might impact Osmotic pressure and therefore drug release rates.

- Simple in vitro dissolution test results were not considered sufficient for granting a biowaiver.

Table 4: Post-Notice of Compliance (NOC) Changes: Quality Document [2]

\section{Appendix 6: Changes to Excipients}

\begin{tabular}{|l|c|}
\hline \multicolumn{1}{|c|}{ Excipient } & $\begin{array}{c}\text { Percent excipient }(w / w) \text { out of total target } \\
\text { dostage form core weight }\end{array}$ \\
\hline Filler & \pm 5.0 \\
\hline Disintegrant & \pm 3.0 \\
\hline Starch & \pm 1.0 \\
\hline Other & \pm 0.5 \\
\hline Binder & \\
\hline Lubricant & \pm 0.25 \\
\hline Ca or Mg Stearate & \pm 1.0 \\
\hline Other & \pm 1.0 \\
\hline Glidant & \pm 0.1 \\
\hline Talc & \pm 1.0 \\
\hline Other & \\
\hline Film Coat 1 & \\
\hline \multicolumn{2}{|l}{$\begin{array}{l}\text { Where the film coat is for appearance only and not intended affect the release rate or stability } \\
\quad \text { characteristics of the drug. }\end{array}$} \\
\hline
\end{tabular}

\section{EXAMPLE 2}

Desvenlafaxine Controlled Release tablet $50 \mathrm{mg}$ and $100 \mathrm{mg}$ (biolot)

- The $50 \mathrm{mg}$ tablet had more of MCC added to the formulation (multifunctional excipient)

- The $50 \mathrm{mg}$ strength had added MCC extra-granularly while for the $100 \mathrm{mg}$ strength no MCC was added extra-granularly.

Risks:

- Distinctly different formulations.

- Impact of MCC (amount and extra granular) on the performance of the MR tablet cannot be predicted.

- In vitro dissolution results not sufficient to justify Biowaivers.

\section{EXAMPLE 3}

Quetiapine Controlled Release tablets $150 \mathrm{mg}, 200 \mathrm{mg}$ (biolot), $300 \mathrm{mg}$ and $400 \mathrm{mg}$.

- Common blend was used for all strengths.

- The release rates were expected to be similar for all strengths.

But different dissolution specifications were proposed for each strength. 
- Only $150 \mathrm{mg}$ and $300 \mathrm{mg}$ qualified for the biowaiver request.

- The $400 \mathrm{mg}$ was withdrawn.

- Subsequently, a supplement for the $400 \mathrm{mg}$ was submitted.

- The formulation remained unchanged, but changes to the manufacturing process were made to achieve similar release profiles to the original biolot $(200 \mathrm{mg})$.

- This could have been done earlier during product development.

\section{EXAMPLE 4}

Valsartan /HCTZ Tablets

- Biostudy strength: $160 \mathrm{mg} / 25 \mathrm{mg}$

- Biowaiver strengths: $320 \mathrm{mg} / 12.5 \mathrm{mg}, 320 \mathrm{mg} / 25 \mathrm{mg}$

- Non proportional formulations: additive effect of all excipients changes $>5.0 \%$; disintegrant $>1.0 \%$.

- Lack of supporting information and justification.

- A biowaiver was not granted and a Notices of Deficiency was issued.

\section{EXMPLE 5}

Pantoprazole DR tablets

- 2 strengths: $20 \mathrm{mg}$ and $40 \mathrm{mg}$ (biolot)

- Cores were directly proportional.

- Coating components were not proportional by weight. Risks: Inadequate enteric coating thickness could also impact drug release. Solution:

- Enteric coating was based on surface area/volume ratio. Two strengths had similar dissolution profiles. Biowaiver was granted.

The following Conclusions were presented:

- Proportionality based biowaivers should satisfy both "proportional formulation" and "proportional performance" criteria.

- When formulation proportionality is not met there is insufficient justification that can be used in place of a biostudy. In such instances it becomes difficult to grant a biowaiver.

This is because false positive in vitro results might show that two strengths are similar, but performance differences cannot be ruled out.

\section{The FDA Perspective of Biowaivers - Examples and Challenges}

Kimberly Raines, Ph.D. Lead Pharmacologist, Quality Assessment Lead (Acting), Center for Drug Evaluation and Research/Office of Pharmaceutical Quality/ Office of New Drug Products/Division of Biopharmaceutics III.

In the US the Code of Federal Regulations is the governing law for the FDA: According to 21 CFR 320.22; FDA shall waive the requirement for the submission of evidence obtained in vivo measuring the bioavailability or demonstrating the bioequivalence of drug products. A drug product's in vivo bioavailability or bioequivalence may be considered self-evident based on other data in the application if the product meets the defined criteria. And 21 CFR 320.24(b) states: 
In vivo and in vitro approaches (as defined), are in descending order of accuracy, sensitivity, and reproducibility, are acceptable for determining the bioavailability or bioequivalence of a drug product.

This presentation refers to either the decision to waive an in vivo BE requirement under 21 CFR 320.22 or the decision to accept in vitro BE data in accordance with 21 CFR 320.24(a) as a "biowaiver".

Proportional Similarity definition of the FDA considers:

1. All active and inactive ingredients are in exactly the same proportion between different strengths (e.g., a tablet of 50-mg strength has all the inactive ingredients, exactly half that of a tablet of 100-mg strength, and twice that of a tablet of 25-mg strength).

2. For high-potency drug substances (where the amount of active drug substance in the dosage form is relatively low),

a. the total weight of the dosage form remains nearly the same for all strengths (within $\pm 10 \%$ of the total weight of the strength on which a BE was performed),

b. the same inactive ingredients are used for all strengths, and

c. the change in any strength is obtained by altering the amount of the active ingredients and one or more of the inactive ingredients.

3. Bilayer tablets are considered to be one formulation even though they consist of two separate layers with different compositions.

a. In assessing the proportional similarity of the different strengths, all components of both layers should be proportionally similar. The fact that only one layer is proportionally similar and the other is not clearly indicates that the products (whole tablet) are not proportionally similar.

b. This is relevant because there can be interactions between the different tablet layers, which can differ across different strengths because of the different size of the layers and the varying amounts of excipients present in each layer.

Cases when FDA was/is granting biowaivers:

A. Self-evident Bioequivalence or Bioavailability:

1. Parenteral solutions: must contain the same active and inactive ingredients in the same amounts as the Reference Listed Drug qualitatively (Q1) and quantitatively (Q2).

2. Product administered by inhalation as a gas $\&$ contains an active ingredient in the same dosage form as Reference Listed Drug.

3. Oral solutions, solutions for application to the skin, elixir, syrup, tincture, a solution for aerosolization or nebulization, a nasal solution: an active drug ingredient in the same concentration and dosage form as Reference Listed Drug; and contains no inactive ingredient or other change in formulation from Reference Listed Drug that may significantly affect absorption of the active drug ingredient that are systemically absorbed, or that may significantly affect systemic or local availability for products intended to act locally.

B. A drug product is in the same dosage form, but in a different strength, and is proportionally similar in its active and inactive ingredients to another drug product for which the same manufacturer has obtained approval, and the Bioavailability of this other drug product has been measured.

1. The applicant submits evidence showing that both drug products are proportionally similar in their active and inactive ingredients;

2. Both products meet an appropriate in vitro test approved by FDA

C. Drug Efficacy Study Implementation (DESI)

In vivo Bioequivalence studies can be waived for solid oral dosage forms that meet these criteria:

1. Approved before 1962 in the US 
2. Determined to be effective for at least one indication in a DESI notice or which is identical, related, or similar to such a drug product

3. No Bioequivalence problems

4. Dissolution data must be acceptable and similar to reference profiles. If there is a USP dissolution method available, then dissolution testing data using USP method may be adequate for the submission. When there is no USP dissolution method for the product but there is a FDA-recommended method, dissolution testing using the FDA-recommended method may be adequate.

D. BCS Biowaiver

1. In vivo BE studies can be waived for IR solid oral dosage forms based on the Biopharmaceutics Classification System (BCS). [14]

2. The guidance updated May 2015 includes biowaiver extension to BCS class 3 drug products and additional modifications, such as criteria for high permeability and high solubility for BCS class 1 drug products. [18]

E. When are Biowaivers granted for Modified Release Products?

1. Biowaivers can be granted if an In Vivo / In Vitro Correlation (IVIVC) is established. [16] The in vitro dissolution method must be predictive and a surrogate for in vivo testing.

Examples are: SUPAC-MR Level 3 manufacturing site changes/release and non-release controlling excipient changes, process changes; [26] Biowaivers of lower Strengths (compositionally proportional/qualitatively the same, similar in vitro dissolution profiles, same release mechanism) and approval of new strengths. However, Level C correlations with as single point correlation are not suitable as IVIVC for biowaivers.

Also, it is not necessary to conduct in vivo studies on all strengths of a MR dosage form. The sponsor may deem other strengths bioequivalent to a corresponding reference strengths when:

1. In vivo Bioequivalence is acceptable for one strength (usually highest)

2. Each strength of a capsule consists of same "fill", differing only in the amount of fill or strengths are proportionally similar in case of tablets.

3. In vitro dissolution test results are acceptable

Dissolution of all strengths in media of at least $3 \mathrm{pH}$ values (e.g., 1.2, 4.5, 6,8) in case of tablets must be tested.

F. When are Biowaivers submitted for New Drug Applications?

1. If a formulation for a new drug product has changed. Differences in formulation and/or manufacturing between proof-of-principle (Phase II) formulations and pivotal formulations (Phase III). However, an in vivo bioequivalence study to the ultimate commercial formulation has to be demonstrated (bridging).

2. Line extensions, e.g., new strengths or new formulations for a new patient population

3. Post-approval changes, including changes of the manufacturing formula, in the manufacturing process, in excipients, in manufacturing sites and/or equipment.

The regulatory overview was followed by case studies.

Case study 1: Biowaiver of lower strength lacking proportionality

The following background information is given: The product is an Extended-Release Formulation containing a BCS Class II drug which converts from amorphous to a crystalline state. This could adversely impact the product performance. 
Case: The Applicant included a biowaiver request for the requirement to submit data from in vivo BA/BE studies for the $1 \mathrm{mg}$ and $0.75 \mathrm{mg}$ strengths of the proposed formulations. However, since the Applicant used different grades of Hypromellose for the $4 \mathrm{mg}$ strength, the formulations of the lower 1 $\mathrm{mg}$ and $0.75 \mathrm{mg}$ strengths, when they compared to the formulation of the $4 \mathrm{mg}$ strength, are not proportionally similar in their active and inactive ingredients.

CONCLUSION: Support approval of the $1 \mathrm{mg}$ and $0.75 \mathrm{mg}$ lower strengths as follows:

1. For the $1 \mathrm{mg}$ strength, a BE study comparing the $4 \mathrm{mg}$ and $1 \mathrm{mg}$ strengths was conducted. The study results support the approval of the $1 \mathrm{mg}$ strength.

2. For the $0.75 \mathrm{mg}$ strength, a biowaiver was requested. The following data were provided to support the approval of the biowaiver request for the $0.75 \mathrm{mg}$ :

- BE study for the $1 \mathrm{mg}$,

- Information demonstrating the proportionality between the composition of the $1 \mathrm{mg}$ and $0.75 \mathrm{mg}$ formulations

- Comparative dissolution profiles between the $1 \mathrm{mg}$ and $0.75 \mathrm{mg}$ with statistical $\mathrm{f} 2$ testing $(>50)$ indicating similarity between these strengths

Case study 2: 505(b)(2) submission (Hybrid between an ANDA [505(j)] and full NDA [505(b)(1)]) including a biowaiver for an oral solution citing a non-listed drug.

The following background information is given: The proposed drug product is an oral solution containing a drug substance known as a beta-adrenergic blocking agent. The submission makes reference to a compounded syrup (syrup is not a listed drug) of two FDA approved products, syrup 5 $\mathrm{mg} / \mathrm{mL}$ compounded from $120 \mathrm{mg}$ tablets. The API is considered highly soluble. The oral Bioavailability following administration of the tablets was $90-100 \%$. Tablets are rapidly dissolving (e.g. $>80 \%$ in $15 \mathrm{~min})$.

CASE: Applicant performed tablet dissolution and disintegration studies on the FDA-approved $120 \mathrm{mg}$ tablets to determine if there might be a significant lag in absorption. The tablets dissolved $(>90 \%)$ in less than 10 min using $0.1 \mathrm{~N} \mathrm{HCl}, \mathrm{pH} 4.5$ acetate buffer, and $\mathrm{pH} 6.8$ phosphate buffer, paddle, $50 \mathrm{rpm}$. All tablets were completely disintegrated within $5 \mathrm{~min}$ for all media tested. These data indicate rapid dissolution and therefore, no potential lag time in absorption (e.g. same Tmax for the tablet vs. solution). The labeling statement for the compounding syrup: "This compounding procedure results in a solution containing $5 \mathrm{mg} / \mathrm{mL}$. The fine solid particles are the water-insoluble inactive ingredients of the tablets." The Applicant prepared an extemporaneous suspension (in triplicate) according to the label instructions. For all tested samples the concentration ranged from $4.96-5.03 \mathrm{mg} / \mathrm{mL}$ ) indication the presence of a true solution. The acceptability of the biowaiver request for the proposed oral solution, $5 \mathrm{mg} / \mathrm{mL}$ referencing a non-listed drug failing to satisfy the criteria for a waiver of evidence of in vivo bioavailability under 21 CFR 320.22 (a), (b). In accordance to 21 CFR 320.22 (b) (3), the criteria to support the biowaiver for an oral solution are summarized below:

1. The proposed drug product is an oral solution

2. Contains an active ingredient (s) in the same concentration and dosage form as an FDAapproved reference product

3. Contains no inactive ingredients or change in formulation that may significantly affect absorption of the active ingredient

CONCLUSION: Support approval of the biowaiver for the oral solution was based on the following:

1. Active ingredient is considered a highly soluble drug substance.

2. The oral BA following administration of the tablets was $90-100 \%$.

3. Tablets are rapidly dissolving (e.g. when 85 percent or more of the labeled amount of the drug substance dissolves within 30 minutes). 
4. Under these circumstances one can assume that the PK of active administered as a solution will be very similar to that following the tablet administration.

Case Study 3: Lower strength biowaiver lacking dissolution profile similarity

The following background information is given: Plans to develop $5 \mathrm{mg}$ and $10 \mathrm{mg}$ IR tablets as a weight multiple of the $15 \mathrm{mg}, 20 \mathrm{mg}, 30 \mathrm{mg}$ and $40 \mathrm{mg}$ tablets (proportionally similar in composition). Given this approach and based on previously provided data on the $15 \mathrm{mg}, 20 \mathrm{mg}, 30 \mathrm{mg}$ and $40 \mathrm{mg}$ tablets, the Applicant concluded in vitro dissolution and stability data would support review and approval of these lower dose strengths, without additional clinical data.

CASE: If $f 2$ testing fails to justify/support the approval of these strengths how can the biowaiver be supported? Lack of similarity is demonstrated between lower and higher strengths. $5 \mathrm{mg}$ and $10 \mathrm{mg}$ tablets were Bioequivalent to the $20 \mathrm{mg}$ tablet which has a slower dissolving profile.

CONCLUSION: A biowaiver was NOT granted. The dissolution data and additional bioequivalence study provided supported the approval of the 5 and $10 \mathrm{mg}$ strengths.

Case Study 4: Approval of lower strength biowaiver lacking dissolution profile similarity

The following background information is given: The Applicant requested biowaiver for the lower strength (75mg) and to-be-marketed scored $150 \mathrm{mg}$ tablets within a 505(b)(2) submission for a BCS Class I drug product. Bioequivalence was demonstrated between 150mg unscored tablet (Clinical Trial Formulation) and the listed product. Additionally, a comparative bioavailability study of the $150 \mathrm{mg}$ capsules demonstrated equivalency to the listed drug product under fed conditions. However, dissolution was not similar between (a) $75 \mathrm{mg}$ and Clinical Trial Formulation; and (b) Clinical Trial Formulation vs. to-be-marketed formulation.

\title{
CONCLUSION:
}

A biowaiver was granted although dissolution dissimilarity based on the totality of the available PK and clinical information and clinical risk-benefit assessment.

The talk concluded that an improved success of Biowaivers is bases on the approach of Integrated Quality Risk-Based Assessment. This requires that an appropriate dissolution test method was selected based on physicochemical, in vitro and in vivo characteristics of drug and drug product. The utilization of technology advances can be used to provide in vitro / in vivo relationships based on the performance of individual dosage form units. It is advisable for sponsors to early communicate with the agency e.g. arranging for an End of Phase 2 meeting between the sponsor and FDA Biopharmaceutics and CMC staff to discuss the biowaiver development plans.

\section{Biowaivers: Options to Consider}

\author{
Mathi Mathivanan PhD., Director, Regulatory Affairs, Teva Canada
}

There are some critical success factors and options to consider before filing for a biowaiver. You need to have the Right product at the first time. This requires a sound formulation strategy (compliance with Health Canada's Policy on Bioequivalence of Proportional Formulations). Convincing in vitro support data with sound experimental design (solubility for drug substance and drug release from drug product). A comprehensive regulatory strategy which uses Health Canada's policies, guidance documents, record of proceedings of Scientific Advisory Committee on Pharmaceutical Sciences and Clinical 
Pharmacology (SAC-PSCP) and other documents such as Q \& A publications. Also being aware of requirements of other regulatory authorities like EMA and FDA.

Health Canada's Policy on Proportional Formulations outlines allowable formulation differences for multiple strengths of a drug product without a need for conducting biostudies for all strengths. To qualify for a biowaiver for a proportional formulation, the total additive effect of all excipient changes should not be more than $5.0 \%$.

An exemption from $5.0 \%$ is allowed under in some cases. For example, the Health Canada guidance on Bioequivalence of Proportional Formulations sates [6]:

"If different strengths have differences in the proportion of ingredients which exceed those in Table, but within the progression of strengths the changes are incremental, a comparative bioavailablity study is required on the lowest and highest strengths. Incremental changes are those in which the proportion of ingredients increase or decrease successively from the lowest to the highest strengths in the range"

Therefore, reliable API solubility data is critical to develop a dissolution method with acceptable sink conditions. Factors Influencing Solubility are substrate/ structure e.g. acidic/basic/neutral, its $\mathrm{pKa}$, temperature, solvent $\mathrm{pH}$ and use of surfactant. Sink conditions are defined as "maintaining a dissolution medium volume that is at least 3 times greater than the saturation volume of the drug substance."

EMA states:

4.2.2 In vitro dissolution tests in support of biowaiver of strengths:

"At pH values where sink conditions may not be achievable for all strengths in vitro dissolution may differ between different strengths. However, the comparison with the respective strength of the reference medicinal product should then confirm that this finding is drug substance rather than formulation related. In addition, the applicant could show similar profiles at the same dose (e.g. as a possibility two tablets of $5 \mathrm{mg}$ versus one tablet of $10 \mathrm{mg}$ could be compared)."

The SAC-PSPC stated in their meeting from June $24^{\text {th }} 2013$ on the question: "Significance of a f2<50 for a lower strength in comparison to the higher strength when all strengths meet sink conditions and all strengths dissolve in less than 30 minutes. Can bioequivalence of the higher strength be extrapolated to the lower faster dissolving strength based on only one $\mathrm{pH}$ ?"

"Yes, as long as it is shown that the Canadian Reference Product exhibits the same dissolution trends in the same media."

Another example is the Questions and Answers from May 23 ${ }^{\text {rd }} 2007$ :

Q1): What is the recommended development data for a dissolution/drug release method?

A): The method development information should include a rationale for choosing the particular apparatus type (paddle, basket, peak vessel etc.), dissolution medium (volume, temperature, $p H$ ), operating conditions (rotation speed), and should establish sink condition (i.e. dissolves $>3$ times the amount of drug). When a surfactant is used in the dissolution medium a rationale should be provided to justify the choice of the surfactant and its concentration. Evidence that the method is discriminatory could also be included in the method validation section.

This was confirmed by the SAC-PSPC; the proceedings from June 25, 2013 state:

Q2: Is it acceptable to use a surfactant in the dissolution medium with a proper justification? A; Yes, as long as the discriminatory power is maintained.

Q3: If yes, what data should be provided to justify the use, and the proposed level of surfactant? A: The principle is that the discriminating power of the dissolution method must be demonstrated, and the lowest level of surfactant should be defined to achieve API solubility. For all dose strength, the same driving force i.e. constant sink conditions should be maintained by adjusting the level of surfactant and the ionic strength. 
Q4: Is it acceptable to compare strengths using different levels of surfactants for the purpose of a biowaiver e.g. FDA QC method?

A: Yes

Q5: Is it meaningful to compare multiple tablets of on strength in the same dissolution vessel to one tablet of the other strength e.g. $2 X 5 \mathrm{mg}$ v.s. $1 X 10 \mathrm{mg}$ ?

A: There should be no reason to do this comparison, considering the response to question 3. However, there might be exceptions that would require a justification.

A historical example was presented. Here testing of multi units $v s$. single units of a biolot was not performed. Also, a comparative dissolution vs. the Canadian Reference Product was not considered. Surfactant concentration appropriate to the tablet strength was not accepted. Hence, bio studies were conducted for $20 \mathrm{mg}$ and $5 \mathrm{mg}$ strengths. For the two non-bio strengths (10 and $2.5 \mathrm{mg}$ ), biowaivers were granted.

An alternate option for Biowaiver was discussed based on the Bioequivalence of Proportional Formulations Policy (1996) [6]: "The comparative dissolution profiles should be determined using a validated QC method. In this regard, a validated method is one that has been demonstrated to be sensitive to changes in formulation and manufacturing, including the physical-chemical attributes of formulation ingredients, as documented in method development studies. In the absence of a validated method, the comparative dissolution profiles should be determined in at least three (3) media within the physiological range ( $\mathrm{pH} 1$ - 7.5), e.g., water, $0.1 \mathrm{~N} \mathrm{HCl}$, and pharmacopeial buffer media at pH 4.5, 6.5 or 7.5. One dissolution medium should be that described in the USP or BP monograph, if one exists."

For immediate release products, if the strengths arise from a common blend and the same manufacturing process and the highest strength is bioequivalent or meets the current requirements for incremental proportionality (highest and lowest strength are bioequivalent to the reference), a waiver is acceptable as long as dissolution profiles are comparable in at least one justified discriminatory method.

If strengths are not coming from the same blend but conform to table 1 of the TPD policy on bioequivalence of proportional formulation and if the bio-study is only done on the highest strength: A biowaiver may be acceptable as long as there are comparable dissolution profiles bases upon two justified discriminatory methods example : different $\mathrm{pH}$ conditions or different biorelevant media.

Today industry has several options to consider if the API solubility is not promising to seek a biowaiver through conventional $\mathrm{f} 2$ approach.

Testing of multi units $v s$. single unit, comparing dissolution profile of test products to the Canadian Reference Product, changing surfactants - type and concentration (right amount for the right strength) and formulating a sound submission strategy (based on Health Canada's policies/guidelines, SAC-PSCP recommendations, Q \& A documents, EMA and FDA recommended options.

Globalization is a new reality for pharmaceutical industry and regulatory convergence is on the horizon.

\section{Biowaivers in the innovator industry}

Barbara M. Davit, PhD, Anita Laloo, Shrinivas Murti, Merck @ Co. USA

A waiver of in vivo bioavailability and/or bioequivalence studies is intended to apply to subsequent in vivo bioavailability and/or bioequivalence studies of formulations after the initial establishment of the in vivo bioavailability of IR dosage forms during the IND period, and in vivo bioequivalence studies of IR dosage forms in ANDAs. 
The FDA BCS Guidance revision 1 specifies: “Appropriate bridging of a test formulation/drug product to a reference formulation/drug product by in vitro studies (comparative dissolution) in place of in vivo studies $(B A / B E)$ "

Biowaivers are used when multiple strengths are in development or an additional strength is added post-approval. Biowaivers are thus a mechanism whereby bridging between strengths can be accomplished via in vitro studies. As one example, if it is necessary to encapsulate a comparator commercial product for blinding, in vitro studies can be used to bridge the over-encapsulated clinical trial materials with the commercial product before the clinical trial takes place. For post approval changes in drug product composition SUPAC Level 1 [1] (minor) or 2 (moderate) changes can be seen as "automatic" biowaivers. A SUPAC Level 3 (major) change must be a "justified" biowaiver and a SUPAC MR (major) biowaivers depend on an IVIVC. [16] The discriminatory power of the dissolution medium must be demonstrated. An IVIVC at a higher strength can be applied to lower strengths provided the same release mechanism is used, a similarity of dissolution profiles was established, and the formulations are proportional to each other. If an IVIVC could not be established, post approval changes must be bridged through a BE study. Ideally an IVIVC (Level A) should be developed prior to Phase III to support registration and post approval changes. Additional guidance can be found in the following respective

Table 5: considerations for biowaivers:

\begin{tabular}{|l|l|}
\hline Technical consideration & $\begin{array}{l}\text { Formulations/strengths amenable to } \\
\text { meaningful in vitro comparison that is } \\
\text { supportive of in vivo bridging studies. }\end{array}$ \\
\hline Regulatory consideration & $\begin{array}{l}\text { Guideline criteria for biowaivers must be } \\
\text { applicable to the product, dosage form or } \\
\text { drug substance. }\end{array}$ \\
\hline Ethical consideration & $\begin{array}{l}\text { Fewer human studies will expose healthy } \\
\text { subjects to pharmacologically active } \\
\text { compounds. }\end{array}$ \\
\hline Risk consideration & $\begin{array}{l}\text { Pharmacokinetic variability might not be } \\
\text { related to absorption processes but to } \\
\text { metabolic or other factors. }\end{array}$ \\
\hline Other considerations & $\begin{array}{l}\text { The number of subjects might be large this } \\
\text { will need more resources and increase the } \\
\text { cost. }\end{array}$ \\
\hline
\end{tabular}

Agencies Guidelines:

- $\quad$ EMA MR Guidance July 1999 [27]

- $\quad$ EMA Draft MR Guidance update March 2013 [28]

Biowaivers for fixed-dose combinations (FDCs) present unique challenges. FDCs are developed because they present several advantages. They may have a greater efficacy due to additive or synergistic effect of each active. They offer the possibility of reduced doses of each active ingredient. 
They might decrease the occurrence of side effects. They increase patient compliance trough convenience of a single unit. This can reduce copay-costs to the patient.

Table 6: Common FDC issues when combining APIs with unique properties

\begin{tabular}{|l|l|}
\hline $\begin{array}{l}\text { Highly variable drugs } \\
\text { (intra-subject variability }>30 \%)\end{array}$ & Atorvastatin, pioglitazone, niacin \\
\hline Active metabolite(s) & Simvastatin, losartan, niacin \\
\hline Insoluble molecules (BCS- II/IV) & Molecules with steep pH-solubility profile \\
\hline Non-linear pharmacokinetics & Metformin (500 mg vs. 1000 mg) \\
\hline $\begin{array}{l}\text { Drug-drug interactions } \\
\text { (metabolic) }\end{array}$ & $\begin{array}{l}\text { Extent of clinical drug-drug interaction effect may have very } \\
\text { different impact on mono-therapy product labels vs. risk for } \\
\text { achieving BE between FDC and co-administration }\end{array}$ \\
\hline $\begin{array}{l}\text { Food effect } \\
\text { API-API chemical interactions } \\
\text { in vivo }\end{array}$ & $\begin{array}{l}\text { Oral bioavailability of rifampicin, an anti-TB drug, is } \\
\text { significantly reduced when dosed with isoniazide as co- } \\
\text { administration or in a FDC }\end{array}$ \\
\hline
\end{tabular}

Table 7: Possible challenges for biowaivers

\begin{tabular}{|l|l|}
\hline Composition and/or process & $\begin{array}{l}\text { Potentially increased differences in release and/or absorption; } \\
\text { Potentially enhanced challenge in demonstrating dissolution } \\
\text { similarity }\end{array}$ \\
\hline $\begin{array}{l}\text { Available dose strengths in } \\
\text { different regions }\end{array}$ & $\begin{array}{l}\text { Potentially increased risk to meeting BE; } \\
\text { Mono-therapy metformin in Canada to get } 1000 \mathrm{mg} \text { is 500 x } \\
\text { 2, so a comparison of the highest strength between a } \\
\text { metformin FDC and mono-therapy would involve 1000 mg in } \\
\text { FDC and 500 mg x 2 in mono-therapy }\end{array}$ \\
\hline Drug loading & $\begin{array}{l}\text { Relative increase resulting in formulation, process and } \\
\text { analytical challenges }\end{array}$ \\
\hline Increased tablet size/surface area & $\begin{array}{l}\text { Impact on dissolution particularly when controlled by tablet } \\
\text { erosion }\end{array}$ \\
\hline
\end{tabular}

The product life cycle management has to deal with one product rather than with multiple. However, combining APIs with unique properties can cause them to interact in formulations with each other or excipients. This can impact dissolution behavior of one or more active ingredient. The extent of interaction could vary as a function of dose strength. Therefore, formulation selection is important and biowaivers are more restricted. It is important include plans for biowaivers upfront when developing FDCs. 
Fixed dose combinations add complexity to the bioavailability strategy of each API due to possible interactions between them and limit the feasibility of certain combinations.

Layered tablets may resolve problems with incompatibilities but do not offer any advantages in terms of biowaivers.

Table 8: FDC and number of actives and their strengths

\begin{tabular}{|l|l|}
\hline Regulatory considerations & $\begin{array}{l}\text { Developing FDC strengths to match all approved mono- } \\
\text { therapy combinations; } \\
\text { Source/choice of reference mono-therapy product. }\end{array}$ \\
\hline Market considerations & $\begin{array}{l}\text { Global development requiring different strength } \\
\text { combinations in different markets: enhances biowaiver } \\
\text { strategy complexity; } \\
\text { Metformin strengths in the US: } 500 \mathrm{mg} \text { and } 1000 \mathrm{mg} ; \text { in the } \\
\text { EU: } 850 \mathrm{mg} \text { and } 1000 \mathrm{mg} \text {; in Canada, 500 mg and } 850 \mathrm{mg} ; \\
\text { Potential multiple BE studies to cover all desired markets }\end{array}$ \\
\hline Clinical considerations & Doses for severe renal impairment \\
\hline
\end{tabular}

Table 9: FDC issues for layered tablets

\begin{tabular}{|l|l|}
\hline Advantages & $\begin{array}{l}\text { Alleviates API-API interaction concerns } \\
\text { Salt conversion } \\
\text { Approach to resolving API-excipient incompatibilities } \\
\text { Allows immediate release and controlled release layers in } \\
\text { single unit } \\
\text { Combination of q.d. and b.i.d. dosed drugs }\end{array}$ \\
\hline Disadvantages & $\begin{array}{l}\text { Increased dissolution behavior challenges due to multiple } \\
\text { matrices; } \\
\text { Adds to process scale-up complexity; } \\
\text { No regulatory relief: BE needs to be demonstrated as the FDC } \\
\text { rather than as individual layers }\end{array}$ \\
\hline
\end{tabular}

The FDA has published proportional similarity FDC guidance in the following draft Guidance for Industry: "Bioavailability and Bioequivalence Studies Submitted in NDAs or INDs - General Considerations". [29]

"Bilayer tablets are considered to be one formulation even though they consist of two separate layers with different compositions. In assessing the proportional similarity of the different strengths, all components of both layers should be proportionally similar. The fact that only one layer is proportionally similar and the other is not clearly indicates that the products (whole tablet) are not proportionally similar. This is relevant because there can be interactions between the different tablet layers, which can differ across different strengths because of the different size of the layers and the varying amounts of excipients present in each layer.

Exceptions to the above definitions may be possible if adequate justification is provided and discussed with the appropriate review division."

Note: Each layer should individually meet the conditions of proportional similarity (i.e.,

independently rather than with respect to the total weight / composition of the bilayers combined). 


\section{BE Bracketing:}

Where bioequivalence assessment at more than two strengths is needed, e.g. because of deviation from proportional composition, a bracketing approach may be used. In this situation it can be acceptable to conduct two bioequivalence studies, if the strengths selected represent the extremes, e.g. the highest and the lowest strength or the two strengths differing most in composition, so that any differences in composition in the remaining strengths is covered by the two conducted studies.

\section{Fixed Dose Combinations:}

The conditions regarding proportional composition should be fulfilled for all active substances of fixed combinations. When considering the amount of each active substance in a fixed combination the other active substance(s) can be considered as excipients.

In the case of bilayer tablets, each layer may be considered independently

\section{Formulation and regulatory strategy:}

Both the EMA and FDA BE Guidelines allow Biowaivers for lower dosage strengths provided the conditions of 'proportional similarity' are met.

The current FDA Guidelines do not provide detailed or specific guidance on BE bracketing strategies when 'proportional similarity' across the different dose strengths is not met.

By contrast, the EMA Guideline: "Guideline on the pharmacokinetic and clinical evaluation of modified release dosage forms" [28]

"where bioequivalence assessment at more than two strengths is needed, e.g. because of deviation from proportional composition, a bracketing approach may be used. It can be acceptable to conduct two bioequivalence studies, if the strengths selected represent the extremes, e.g. the highest and the lowest strength or the two strengths differing most in composition, so that any differences in composition in the remaining strengths is covered by the two conducted studies."

\section{Bilayer Tablets}

Biowaivers may be sought for lower dose strengths based on 'dose proportionality', but note the EMA and FDA treat dose proportionality of bilayer tablet systems differently

FDA: "Bilayer tablets are considered to be one formulation even though they consist of two separate layers with different compositions. In assessing the proportional similarity of the different strengths, all components of both layers should be proportionally similar."

EMA: "The conditions regarding proportional composition should be fulfilled for all active substances of fixed combinations. When considering the amount of each active substance in a fixed combination the other active substance(s) can be considered as excipients. In the case of bilayer tablets, each layer may be considered independently..."

\section{Examples:}

1) Agency 1 and Agency 2 require different approaches for using biowaivers for bracketing intermediate strengths of a FDC.

MK-XY is a fixed dose combination with linear PK across therapeutic dosing ranges of API X and API $\mathrm{Y}$, the formulation is not proportionally similar across all 4 strengths; dissolution similarity could be demonstrated between:
- $\quad$ high/high and high/low
- low/high and low/low 
Sponsor proposed the following for two different agencies:

Agency 1: in vivo: High/high and low/low MK-XY strengths

Comparative dissolution profile data for the two middle strengths vs. the proposed highest and lowest strengths

In the above case, Agency 1 considered the FDC as one formulation with four strengths. Proportional similarity is needed across all strengths. This agency indicated it would accept a bracket approach at the extreme strengths.

\section{Agency 2: In vivo: High/low and low/high MK-XY strengths}

Waive the other strengths based on dissolution results, because the formulation is not proportional similar across all four strengths. This agency indicated it would accept a bracketing approach based on strengths with the greatest differences in composition.

\section{2) Biowaiver for clinical blinding}

Over-encapsulation was required in a Phase III study for blinding. A biowaiver can be justified to support blinding. Both formulations exhibit rapid release (> 85\% @ $15 \mathrm{~min}$ and low pH). However, both formulations show slow release for all strengths in surfactant media. The BE risk was assessed to be low. A BE study showed that the low strengths are bioequivalent, but the higher strengths failed BE.

Lessons Learned: Formulations can be very sensitive to small changes in composition as shown by the addition of gelatin capsule around tablet.

\section{3) Development of an IVIVC}

The formulation contained a BCS Class 2 compound. Four different batches were manufactured with different hardness levels to produce distinct dissolution profiles. A BE study showed no differences for AUC but a clear relationship was observed between dissolution rate and $\mathrm{C}_{\max }$. Regulatory agencies accepted such Level $\mathrm{C}$ correlations to define suitable tablet hardness ranges within $\mathrm{C}_{\max }$ limits. Thus, the IVIVC results were useful in developing clinically relevant manufacturing specifications and process controls

\section{Going Forward: Formulation Strategies for Biowaivers}

It is important to consider possible biowaiver strategies in formulation development for fixed dose combinations. Here the entire unit should be compositionally proportional. In the US there is no biowaiver advantage for bilayer technology where one strength is constant and the other is changing; however, in Europe a biowaiver for bilayer tablets is possible on the basis of composition of individual layers. A biowaiver strategy for multiple strengths should consider early communication with the agencies to guide formulation and bridging strategies.

\section{Challenges in Biowaivers: A Health Canada Perspective}

Shereeni Veerasingham, PhD, Bureau of Pharmaceutical Sciences, Therapeutics Products Directorate.

Different scenarios were described to explain the challenges with biowaivers for additional strengths. The presentation excluded BCS-based biowaivers as that was the focus of a prior CSPS workshop.

In general, pharmaceutical development should link in vitro dissolution to in vivo product performance. Under certain conditions, the results of a comparative bioavailability or clinical study can be extrapolated to all strengths in a product series. Considerations for a biowaiver include the pharmacokinetics of the drug, the type of dosage form, manufacturing processes and proportionality of the formulations. Pharmacokinetic characteristics that impact biowaiver data requirements include 
whether the drug displays linear or non-linear pharmacokinetics or exhibits a narrow therapeutic range. In general, comparative bioavailability studies conducted under fasting conditions provide a discriminatory test of product performance. However, studies in the fed state may be acceptable under certain situations $e$.g. serious safety risk with administration of the drug under fasting conditions, when drug concentrations are not reliably measured in the absence of food or when food effects significantly enhance bioavailability. For modified release products, studies conducted under fasted and fed conditions are required.

\section{Appropriate strength for in vivo studies:}

For drugs that display linear pharmacokinetics, preferably the highest strength should be used in comparative bioavailability studies. A lower dose may be employed for safety reasons. An assessment of non-linear pharmacokinetics is considered as a difference in dose-adjusted mean AUC $>25 \%$, and is based on available data, the scientific literature and the Canadian labelling for the drug. For drugs that display non-linear pharmacokinetics with a greater than proportional increase in AUC, at least the highest strength should be tested in vivo. If less than proportional increases in AUC is observed with increasing doses due to saturable absorption, at least the lowest strength (single dose unit) should be used. If limited solubility of the medicinal ingredient results in less than proportional increases in AUC with increasing doses, at least the lowest strength (single unit dose) should be tested under fasted conditions and highest strength should be tested under fasted and fed states.

\section{Biowaivers Based on Proportionality:}

When formulations are proportional or have only minor differences in the proportion of excipients relative to the biobatch strength, the results of an in vivo study can be extrapolated to all strengths in the product series. The requirements to be met are described in the policy: Bioequivalence of Proportional Formulations - Solid Oral Dosage Forms (1996). The criteria include both proportionality of formulations as well as comparative dissolution profiles. Additional recommendations were given by the Scientific Advisory Committee on Pharmaceutical Sciences and Clinical Pharmacology (SACPSPC) in 2013, for products containing low solubility drug substances and fixed dose combinations with drug substances of differing solubility. Formulations are considered to be proportional when each strength contains the same ingredients in the same proportion. Differences in the proportion of excipients are considered to be minor when the differences in amounts for excipients of particular functions are within the limits described in Table 1 of the aforementioned Health Canada guidance, and the total additive effect of changes are not more than 5.0\%. Current practice includes a requirement that multifunctional excipients meet the most conservative applicable limit for its defined functions. A bracketing approach with in vivo equivalence studies conducted on the highest and lowest strengths may be used for incremental changes in formulation that exceed the specified limits. For modifiedrelease products, no changes in the proportions of release controlling excipients are permitted. The proportion of each ingredient is calculated as a percentage $(\mathrm{w} / \mathrm{w})$ of the total core weight. However, in some cases such as delayed-release products with enteric coatings, the proportions of coating components are based on the tablet surface area.

\section{Comparative Dissolution Testing:}

A validated QC method that is sensitive to modest changes in formulation, including physical-chemical attributes of the ingredients, and manufacturing process should be used for comparative dissolution testing. Alternatively, multi-media dissolution methods that include at least 3 media covering the physiological range $(\mathrm{pH} 1.2-6.8)$ may be used. The selected media should emphasize possible differences between the product strengths, e.g. $\mathrm{pH}$ conditions close to the $\mathrm{pKa}$ value of the drug. Similarity between the dissolution profiles should be demonstrated. A determination of the modelindependent similarity factor (f2) is recommended (Appendix 5 of Health Canada's Post-Notice of Compliance Changes: Quality Document). [2] 


\section{Example 1}

The first example described an immediate release tablet, formulated with incremental changes in the proportion of filler between the four strengths in the product series $(10,20,30$ and $40 \mathrm{mg})$. The proportion of filler decreased successively across the strengths with up to $15 \%$ difference, which exceeds the 5\% limit specified for minor changes. A bracketing approach is required, with in vivo equivalence studies conducted on the lowest and highest strengths. A further consideration would be the pharmacokinetics of the drug. In this example, limited drug solubility results in less than proportional increases in AUC with increasing doses. A biowaiver of the intermediate strengths would therefore require a comparative bioavailability study conducted under fasting conditions employing the low strength (10 mg, single unit dose). In addition, comparative bioavailability studies should be conducted under fasted and fed conditions with the high strength $(40 \mathrm{mg})$.

\section{Fixed Dose Combination Products:}

In general, for fixed dose combination products, each API is considered separately in relation to the excipients. The proportion of an excipient is based on the dosage form core weight of the tablet/capsule and includes all drug substances. For layered tablets, formulation layers are generally considered independently. The assessment is performed on a case-by-case basis and depends on the formulation design. In some cases, multiple matrices containing APIs of differing solubilities may introduce dissolution challenges. In vivo equivalence studies to bracket fixed dose combination formulations may be based on the extremes in formulation composition and/or strengths. For drug products where at least one API is of low solubility, if formulations are proportional and ratios of APIs change, the SAC-PSPC recommended in vivo studies bracketing of the extremes of the API ratios.

\section{Example 2}

This example considered a fixed dose combination, immediate release layered tablet which was developed to match the approved mono-therapy combinations. The product series included four strengths, FDC X/Y: 40/5, 40/10, 80/5 and 80/10 mg. The solubility of the APIs over the physiologic range differs: API X exhibits low solubility and API Y exhibits high solubility. The extremes of the API ratios are represented by the $80 / 5$ and $40 / 10$ mg strengths. Further, API X exhibits non-linear pharmacokinetics over the therapeutic dose range, with greater than proportional increases with increasing doses, whereas API Y exhibits linear, dose- proportional kinetics. Based on the formulation design, the strengths are considered proportional with respect to each API layer: API X layer is directly proportional; API Y layer has minor differences relative to the biobatch. Considering the pharmacokinetics of the APIs, studies should be conducted with the higher strength for API X and preferably, the higher strength for API Y, i.e. FDC 80/10 mg versus co-administered respective strengths of $\mathrm{X}$ and $\mathrm{Y}$ to bridge the formulations. Due to differences the assessment of proportionality of fixed dose combination products across different regulatory agencies, studies conducted with the low/low and high/high API combinations were provided i.e. studies with FDC 40/5 and 80/10 mg strengths. Biowaivers were granted for the $40 / 10$ and $80 / 5 \mathrm{mg}$ strengths based on proportional formulation to the biobatches and a demonstration of similar multi-media dissolution profiles. This example highlights that the assessment of proportionality of a layered tablet impacts the choice of strength(s) to be tested in equivalence studies.

\section{Example 3}

Capecitabine capsules are approved as 150 and $500 \mathrm{mg}$ strengths; the drug is considered highly soluble in the $\mathrm{pH}$ range, $2.5-6.8$ with the lowest solubility at $\mathrm{pH} 2.5$ (Dose/Solubility Volume of $246 \mathrm{~mL}$ ). However, capecitabine is unstable at highly acidic conditions of $\mathrm{pH} \mathrm{1.2,} \mathrm{which} \mathrm{limits} \mathrm{the} \mathrm{possibility} \mathrm{of}$ a BCS-based biowaiver. The pharmacokinetics are linear over the therapeutic dose range. In this example, bioequivalence was demonstrated for Capecitabine $500 \mathrm{mg}$ capsules, with a biowaiver request for the proportionally formulated $150 \mathrm{mg}$ strength. Dissolution data for $3 \times 150 \mathrm{mg}$ in comparison to 1 x $500 \mathrm{mg}$ demonstrate similar profiles in four media across the physiological $\mathrm{pH}$ range $(\mathrm{pH} 1.2-6.8$, 
$\mathrm{f}_{2}>50$ ). However, data provided for single units indicate similar dissolution in $0.1 \mathrm{~N} \mathrm{HCl}, \mathrm{pH} 2.1$ and acetate buffer, $\mathrm{pH} 4.5$ ( $\left.\mathrm{f}_{2}>50\right)$, but the lower strength dissolves faster in phosphate buffer, $\mathrm{pH} 6.8$ and the QC medium, water $\left(\mathrm{f}_{2}<50\right)$. Considerations for a biowaiver of the lower strength included: the formulations of the strengths are directly proportional, with the same qualitative composition as the innovator product; the $150 \mathrm{mg}$ capsules exhibit more than $85 \%$ dissolved within 30 minutes in all media; in all comparisons, the $150 \mathrm{mg}$ dissolves faster than the $500 \mathrm{mg}$ strength and the same dissolution trends are noted for the reference product. Therefore, the differences in dissolution are not expected to be clinically relevant. The biowaiver was granted, in line with recommendations of the SAC-PSPC for a lower, faster dissolving strength.

\section{Example 4}

Ziprasidone Capsules are marketed as 20, 40, 60 and $80 \mathrm{mg}$ strengths, and the lowest strength is employed in bioequivalence studies due to safety considerations (risk of QT prolongation). Ziprasidone is poorly soluble in the physiological $\mathrm{pH}$ range of $1.2-6.8$. The SAC-PSPC recommendations highlight that "Products containing a low solubility Active Pharmaceutical Ingredient (API) should generally not be eligible for a biowaiver for a higher strength than employed in a bioequivalence study due to a risk of inequivalent therapeutic effects." However, Ziprasidone is labeled to be administered with food. Administration with food increases ziprasidone bioavailability up to 2-fold and the pharmacokinetics are linear within the therapeutic dose range. Generally, linear pharmacokinetics reduces the risk that is inherent in extrapolating in vivo data obtained for a lower strength of a low solubility drug to higher strengths. In the presented example, a bioequivalence study was conducted with $20 \mathrm{mg}$ in the fed state with a waiver request for the higher strengths. Considerations for the biowaiver were: the strengths are directly proportional in formulation and use a wet granulation manufacturing process which improves homogeneity. Similar multi-media dissolution profiles for the higher strengths versus the biobatch were demonstrated, with adequate justification for the use of surfactant. Commercial scale batches were provided for registration, and the batches exhibit similar multi-media dissolution profiles to that of the biobatch. A biowaiver was granted with restrictive release specifications bench-marked to the biobatch, with consideration given to data from pilot biostudies for formulation variants.

Overall, a risk-based approach to assessing biowaiver requests for additional strengths was emphasized. The examples presented illustrate the factors considered by Health Canada in granting biowaivers.

\section{Bioequivalence and Biowaivers for Modified Release dosage forms}

\section{Laszlo Endrenyi, Ph.D., University of Toronto}

The two guidelines of the European Medicines Agency (EMA) for biowaivers has issued the most detailed guidance on biowaivers. [8]

The general BE guideline, issued in 2010, stated the conditions under which biowaivers could be issued. The more specific guideline for MR drug products, issued later, defined the requirements for granting biowaivers. [28]

Single-dose investigations performed at three dosing strengths under both fasting and fed conditions are required for all MR formulations. With extended (prolonged) release products, multipledose studies are also expected if they exhibit substantial accumulation. However, at the middle and low dosing strengths of single-unit of both delayed- and extended-release formulations, waivers can be granted in single-dose studies, performed under either the fasting or fed condition, which are not recommended by the Summary of Product Characteristics (SmPC) and also at the middle and low dosing strengths of multiple-unit investigations. Waivers can be granted in multiple-dose studies of extended-release (ER) formulations performed at the middle and low strengths. Waivers are not provided at the highest dosing strength. 
The FDA requires, in addition to single-dose studies, also multiple-dose studies when a new extended-release formulation is tested against an approved immediate-release product and also when it is compared with a marketed extended-release formulation used at different dosing intervals. However, multiple-dose investigations are not need if the dosing intervals are the same. Studies should be performed generally at the highest dosing strength. With drugs having nonlinear pharmacokinetics, the investigations should be pursued, at least, at the highest and lowest dosing strengths of the extendedrelease product.

Health Canada does not require multiple-dose studies.

Analyses of in-vitro in-vivo correlations (IVIVC) are needed to support claims of biowaivers. Level A correlation is required which predicts the entire concentration-time profile based on dissolution/drug release data. The IVIVC investigations should be performed in the fasting state even when the Summary of Product Characteristics (SmPC) [30] recommends fed studies. The IVIVC model should be validated.

The bioequivalence of multiphasic MR products was then discussed. Several of these formulations contain two components, one immediate- and one modified-release constituent. FDA has recognized alertly their distinct properties. It issued specific guidance for some products, which were rational and based on science. For instance, the guidance for extended-release (ER) tablets of zolpidem tartrate [31] requires that, in addition to the comparison of the usual metrics, AUC and Cmax, also the partial AUC be evaluated from to $1.5 \mathrm{hr}$ following drug administration and from $1.5 \mathrm{hr}$ to the time of infinity. This expectation is justified by noting that $95 \%$ of the subjects are asleep by $1.5 \mathrm{hr}$ after dosing, and that equivalent sleep maintenance is anticipated thereafter.

The background of extended-release methyl phenidate tablets is even more interesting. The draft guidance of FDA, issued in September, 2012, set the additional requirements of evaluating partial AUCs from 0 to $3 \mathrm{hr}$ post-dosing and from $3 \mathrm{hr}$ to infinity in studies conducted in the fasting state. [32] In investigation performed in the fed state, the additional partial AUCs were to and from $4 \mathrm{hr}$. The rationale was based on the properties of formulation and on features of the multiphasic concentrationtime profile. [33, 34]

The FDA draft guidance was revised in November, 2014. [35] Two additional cut-off times were set at 7 and $10 \mathrm{hr}$ and at 8 and $10 \mathrm{hr}$ for studies conducted in the fasting and fed state, respectively. Additional requirements were announced such as the undertaking of 4-period investigations and the analysis of subject-by-formulation interaction. The reason for the new standards was that post-approval surveillance of two generics, by Kudco and Mallincrodt, had been found to have insufficient effects. [36] Details of the background and new requirements were provided in an FDA 2014 Summary Report. [37]

Comparisons of concentration profiles between various generic ER-MPH tablets and of the reference product (Concerta $\left.{ }^{\circledR}\right)$ indicate large differences among the shapes of the generic profiles. The largest separation between the concentration profiles ranges of the generic and reference products ranges from 3 to 12 hours. This is important and relevant since strong correlation was reported between time courses of concentrations and clinical responses.

In view of the diversity of the generic concentration shapes, it may not be useful to set the same rule for all products on the cut-off times of the partial AUCs. Indeed, the EMA guideline states: "The time point for truncating the partial AUC should be based on the PK profile for the e.g. IR and the MR parts respectively and should be justified and pre-specified in the study protocol." Therefore, the caseby-consideration of the cut-off time for partial AUCs should be entertained. 
Post-Workshop Notes: Since the above workshop date, many further reports have been published that are worthy of mentioning; e.g., those by J. E van Oudtshoorn et al (38) and C. Crane et al (39).

\section{REFERENCES}

1. FDA, Immediate Release Solid Oral Dosage Forms Scale-Up and Postapproval Changes: Chemistry, Manufacturing, and Controls, In Vitro Dissolution Testing, and In Vivo BioequivalenceDocumentation, Center for Drug Evaluation and Research (CDER), Editor 1995, Food and Drug Administration: Rockville.

2. Helath Canada, Post- Notice of Compliance (NOC) Changes: Quality Document 2016, 2016: Ottawa.

3. Leblond, D., et al., In vitro dissolution curve comparisons: A critique of current practice. Dissolution Technologies, 2016. 23(1): p. 14-23.

4. Helath Canada, Biopharmaceutics Classification System Based Biowaiver, 2014: Ottawa.

5. FDA, Guidance for industry: Waiver of in vivo bioavailability and bioequivalence studies for immediate-release solid oral dosage forms based on a Biopharmaceutics Classification System, Center for Drug Evaluation and Research (CDER), 2000.

6. Health Canada, Bioequivalence of Proportional Formulations, 1996: Ottawa.

7. FDA, Draft guidance for industry: Bioavailability and bioequivalence studies submitted in NDAs and INDs - general considerations. Center for Drug Evaluation and Research (CDER), 2014, Food and Drug Administration: Silver Spring, MD.

8. European Medicines Agency, Guideline on the Investigation of Bioequivalence, 2010.

9. FDA, Guidance for Industry; Q8(R2) Pharmaceutical Development, Center for Drug Evaluation and Research (CDER), 2009.

10. Pharmaceutical and Food Safety Bureau, Japan, Guideline for Bioequivalence Studies of Generic Products, 2012.

11. SimulationsPlus. GastroPlus Software. 2017.

12. Almukainzi, M., et al., Disease specific modeling: Simulation of the pharmacokinetics of meloxicam and ibuprofen in disease state vs. healthy conditions. European Journal of Pharmaceutics and Biopharmaceutics, 2016. 100: p. 77-84.

13. Okumu, A., M. DiMaso, and R. Löbenberg, Dynamic dissolution testing to establish in vitro/in vivo correlations for montelukast sodium, a poorly soluble drug. Pharmaceutical Research, 2008. 25(12): p. 2778-2785.

14. Amidon, G.L., et al., A Theoretical Basis for a Biopharmaceutic Drug Classification: The Correlation of in Vitro Drug Product Dissolution and in Vivo Bioavailability. Pharmaceutical Research: 1995. 12(3): p. 413-420.

15. Löbenberg, R. and G.L. Amidon, Modern bioavailability, bioequivalence and biopharmaceutics classification system. New scientific approaches to international regulatory standards. European Journal of Pharmaceutics and Biopharmaceutics, 2000. 50(1): p. 3-12.

16. FDA, Extended Release Oral Dosage Forms: Development, Evaluation, and Application of In Vitro/In Vivo Correlations, Center for Drug Evaluation and Research (CDER), 1997, Food and Drug Administration: Rockville.

17. WHO, Proposal to waive in vivo bioequivalence requirements for WHO Model List of Essential Medicines immediate-release, solid oral dosage forms, in 40th Report, Annex 8 of WHO Expert Committee on Specifications for Pharmaceutical Preparations, WHO, 2006.

18. FDA, Draft Guidance for Industry: Waiver of in vivo bioavailability and bioequivalence studies for immediate-release solid oral dosage forms based on a Biopharmaceutics Classification System, Center for Drug Evaluation and Research (CDER), 2015, Food and Drug Administration: Silver Spring, MD. 
19. Yazdanian, M., et al., The "High Solubility" Definition of the Current FDA Guidance on Biopharmaceutical Classification System May Be Too Strict for Acidic Drugs. Pharmaceutical Research, 2004. 21(2): p. 293-299.

20. Bou-Chacra, N., et al., Evolution of Choice of Solubility and Dissolution Media After Two Decades of Biopharmaceutical Classification System. AAPS Journal, 2017: p. 1-13.

21. WHO, Multisource (generic) pharmaceutical products: guidelines on registration requirements to establish interchangeability. 2015. Technical report 992.

22. WHO, WHO model list of essential medicines. 2015.

23. $\mathrm{Wu}, \mathrm{C.Y}$. and L.Z. Benet, Predicting drug disposition via application of BCS: Transport/absorption/ elimination interplay and development of a biopharmaceutics drug disposition classification system. Pharmaceutical Research, 2005. 22(1): p. 11-23.

24. FDA, Dissolution Testing and Specification Criteria for Immediate-Release Solid Oral Dosage Forms Containing Biopharmaceutics Classification System Class 1 and 3 Drugs, Center for Drug Evaluation and Research (CDER), 2015, Food and Drug Administration: Silver Spring, MD.

25. Uebbing, L., et al., Justification of disintegration testing beyond current FDA criteria using in vitro and in silico models. Drug Design, Development and Therapy, 2017. 11: p. 1163-1174.

26. FDA, SUPAC-MR: Modified Release Solid Oral Dosage Forms Scale-Up and Postapproval Changes: Chemistry, Manufacturing, and Controls; In Vitro Dissolution Testing and In Vivo Bioequivalence Documentation, Center for Drug Evaluation and Research (CDER),1997, Food and Drug Administration: Rockville.

27. European Medicines Agency, Modified release oral and transdermal dosage forms, 1999.

28. European Medicines Agency, Guideline on the pharmacokinetic and clinical evaluation of modified release dosage forms, 2013.

29. FDA, Bioavailability and Bioequivalence Studies Submitted in NDAs or INDs - General Considerations. (CDER), Editor 2014, Food and Drug Administration: Rockville.

30. European Medicines Agency, A guidline on summary of product characteristics (SmPC), E. Commission, 2009.

31. FDA, Draft guidance on Zolpidem Tartrate, Center for Drug Evaluation and Research (CDER), 2012, Food and Drug Administration: Silver Spring, MD

32. FDA, Draft guidance on methylphenidate, Center for Drug Evaluation and Research (CDER), 2012, Food and Drug Administration: Silver Spring, MD.

33. Stier, E.M., et al., Use of partial area under the curve metrics to assess bioequivalence of methylphenidate multiphasic modified release formulations. AAPS Journal, 2012. 14(4): p. 925926.

34. Fourie Zirkelbach, J., et al., Use of partial AUC (PAUC) to evaluate bioequivalence - a case study with complex absorption: methylphenidate. . Pharm Res., 2013. 30(1): p. 191-202.

35. FDA, Draft guidance on methylphenidate, Center for Drug Evaluation and Research (CDER), 2014, Food and Drug Administration: Silver Spring, MD.

36. FDA, Questions and answers regarding methylphenidate extended release tablets (generic Concerta) made by Mallinckrodt and Kudco., Center for Drug Evaluation and Research (CDER), 2014, Food and Drug Administration: Silver Spring, MD.

37. FDA, Summary report: Methylphenidate extended release tablets (generic Concerta) made by Mallinckrodt and Kudco., Center for Drug Evaluation and Research (CDER), 2014, Food and Drug Administration: Silver Spring, MD.

38. Joy Elizabeth van Oudtshoorn, Alfredo García-Arieta, Gustavo Mendes Lima Santos, et al. A Survey of the Regulatory Requirements for BCS-Based Biowaivers for Solid Oral Dosage Forms by Participating Regulators and Organisations of the International Generic Drug Regulators

Programme. J Pharm Pharm Sci, 21 2018, p. 1-142; doi.org/10.18433/J3X93K 
39. Christopher Crane, Gustavo Mendes Lima Santos, Eduardo Agostinho Freitas Fernandes et al, The Requirements for Additional Strength Biowaivers for Immediate Release Solid Oral Dosage Forms in International Pharmaceutical Regulators Programme Participating Regulators and Organisations: Differences and Commonalities. J. Pharm Pharm Sci 22, 2019, P. 365 - 629. doi.org/10.18433/jpps30724 